\title{
Recurrent high-grade glioma surgery: a multimodal intraoperative protocol to safely increase extent of tumor resection and analysis of its impact on patient outcome
}

\author{
Giuseppe Maria Vincenzo Barbagallo, MD, ${ }^{1,2}$ Francesco Certo, MD, PhD, ${ }^{1,2}$ \\ Stefania Di Gregorio, MD,1 Massimiliano Maione, MD,1 Marco Garozzo, MD, ${ }^{1}$ \\ Simone Peschillo, MD, ${ }^{1}$ and Roberto Altieri, MD ${ }^{1-3}$ \\ 'Division of Neurosurgery, Department of Neurosciences, Policlinico "G. Rodolico-S. Marco," University Hospital, Catania; \\ 2Multidisciplinary Research Center on Brain Tumors Diagnosis and Treatment, University of Catania; and 'Department of \\ Neuroscience, University of Turin, Italy
}

\begin{abstract}
OBJECTIVE No consensus exists on the best treatment for recurrent high-grade glioma (HGG), particularly in terms of surgical indications, and scant data are available on the integrated use of multiple technologies to overcome intraoperative limits and pitfalls related to artifacts secondary to previous surgery and radiotherapy. Here, the authors report on their experience with the integration of multiple intraoperative tools in recurrent HGG surgery, analyzing their pros and cons as well as their effectiveness in increasing the extent of tumor resection. In addition, they present a review of the relevant literature on this topic.
\end{abstract}

METHODS The authors reviewed all cases in which recurrent HGG had been histologically diagnosed after a first surgery and the patient had undergone a second surgery involving neuronavigation with MRI, intraoperative CT (iCT), ${ }^{11} \mathrm{C}$ methionine-positron emission tomography $\left({ }^{11} \mathrm{C}-\mathrm{MET}-\mathrm{PET}\right), 5$-aminolevulinic acid (5-ALA) fluorescence, intraoperative neurophysiological monitoring (IONM), and intraoperative navigated ultrasound (iUS). All cases were classified according to tumor functional grade (1, noneloquent area; 2, near an eloquent area; 3, eloquent area).

RESULTS Twenty patients with recurrent HGG were operated on using a multimodal protocol. The recurrent tumor functional grade was 1 in 4 patients, 2 in 8 patients, and 3 in the remaining 8 patients. In all patients but 2, 100\% EOTR was obtained. Intraoperative 5-ALA fluorescence and navigated iUS showed low specificity and sensitivity. iCT detected tumor remnants in 3 cases. Postoperatively, 6 patients (30\%) had worsening neurological conditions: 4 recovered within 90 days, 1 partially recovered, and 1 experienced a permanent deficit. The median Karnofsky Performance Status remained substantially unchanged over the follow-up period. The mean progression-free survival after the second surgery was 7.7 months (range 2-11 months). The mean overall survival was 25.4 months (range 10-52 months), excluding 2 long survivors. Two patients died within 60 days after surgery, and 3 patients were still under follow-up at the end of this study.

CONCLUSIONS This is the first study reporting the integration of neuronavigation, 5-ALA fluorescence, iUS, iCT, ${ }^{11} \mathrm{C}$ MET-PET, and IOM during microsurgical resection of recurrent glioma. The authors believe that the proposed multimodal protocol is useful to increase the safety, effectiveness, and EOTR in patients with recurrent HGG and brain alterations secondary to radio- and chemotherapy.

https://thejns.org/doi/abs/10.3171/2020.10.FOCUS20744

KEYWORDS glioma recurrence surgery; 5-aminolevulinic acid fluorescence; ${ }^{11} \mathrm{C}-\mathrm{MET}-\mathrm{PET}$; intraoperative computed tomography; intraoperative ultrasound

\footnotetext{
ABBREVIATIONS 5-ALA = 5-aminolevulinic acid; ${ }^{11} \mathrm{C}-\mathrm{MET}$-PET = ${ }^{11} \mathrm{C}$-methionine-positron emission tomography; $\mathrm{AA}=$ anaplastic astrocytoma; DES = direct cortical and subcortical electrical stimulation; DTI = diffusion tensor imaging; EOTR = extent of tumor resection; GBM = glioblastoma; GTR = gross-total resection; HGG = high-grade glioma; iCT = intraoperative CT; iMRI = intraoperative MRI; IONM = intraoperative neurophysiological monitoring; iUS = intraoperative ultrasound; KPS = Karnofsky Performance Status; MEP = motor evoked potential; NIHSS = National Institutes of Health Stroke Scale; OS = overall survival; PFS = progression-free survival; SSEP = somatosensory evoked potential; STR = subtotal resection.
}

SUBMITTED August 16, 2020. ACCEPTED October 28, 2020.

INCLUDE WHEN CITING DOI: 10.3171/2020.10.FOCUS20744. 
$\mathrm{G}$ LIOMAS represent a small percentage of tumors worldwide, and $50 \%$ are anaplastic astrocytomas (AAs) or glioblastomas (GBMs), both defined as high-grade gliomas (HGGs) according to the WHO histological brain tumor classification. ${ }^{1}$ Despite a low presentation rate for both tumors, the mean overall survival (OS) rates for AAs and GBMs are 3-5 years and 15-18 months, respectively. ${ }^{2}$ However, different factors influence survival, such as age, Karnofsky Performance Status (KPS) score, and tumor location, invasiveness, and genetic pattern. ${ }^{3}$

Although improvements in glioma treatment have been made over the years, the European Organisation for Research and Treatment of Cancer-National Cancer Institute of Canada trial reported $11 \%$ and $4 \%$ progression-free survival (PFS) rates at 2 and 5 years, respectively. ${ }^{4}$ Therefore, effective management of recurrences is challenging in terms of either early diagnosis of a real recurrence on follow-up MRI or appropriate treatment (radiotherapy and/or chemotherapy with or without surgery). To date, there is no consensus on the protocol for treating recurrent $\mathrm{HGG}$ due to a lack of relevant studies and discordant results.

Extent of tumor resection (EOTR) is a recognized prognostic factor, and a greater EOTR at both the first and the second (i.e., for recurrent tumors) surgery is associated with higher survival rates. ${ }^{5,6}$ To increase the rate of gross-total resection (GTR), new intraoperative tools are under evaluation. For primary HGG surgery, different associations between neuronavigation and one or more of the following tools have been reported in different studies: 5-aminolevulinic acid (5-ALA) fluorescence, intraoperative neurophysiological monitoring (IONM), intraoperative MRI (iMRI), intraoperative CT (iCT), and intraoperative ultrasound (iUS). ${ }^{7-11}$ Conversely, the literature lacks clear data on the role of these tools in recurrent glioma surgery, and even less information is available on their combined use in a multimodal protocol to overcome the intraoperative pitfalls related to previous surgery and radiotherapy.

Here, we report our experience with the integration of multiple intraoperative tools in recurrent HGG surgery, analyzing their pros and cons as well as their effectiveness in increasing EOTR. In addition, we present a review of the relevant literature on this topic.

\section{Methods}

We reviewed our institution's electronic medical charts for appropriate cases. Inclusion criteria were a histopathological diagnosis of HGG after a first surgery, a KPS score $>60$, feasible GTR of recurrent tumor according to preoperative MRI, age $<80$ years, and positive ${ }^{11} \mathrm{C}$-methioninepositron emission tomography $\left({ }^{11} \mathrm{C}-\mathrm{MET}-\mathrm{PET}\right)$ clearly differentiating recurrent pathology from radionecrosis or pseudoprogression. Exclusion criteria were a low KPS score $(\leq 60)$, poor general conditions, and infeasible GTR due to tumor location (i.e., eloquency) or multifocality.

Before surgery, all included patients underwent MRI with the following sequences: T1, T2, volumetric Gdenhanced T1-weighted, FLAIR, diffusion tensor imaging (DTI), and spectroscopy. DTI sequences were elaborated using the StealthViz planning station (Medtronic, USA). All sequences, even 3D reconstructed fiber tracking, were uploaded into the navigation system (S8 StealthStation, Medtronic, USA). Moreover, ${ }^{11}$ C-MET-PET was obtained to study tumor metabolism and used for intraoperative image guidance. ${ }^{11} \mathrm{C}$-MET-PET was also used during followup to differentiate real tumor progression from radionecrosis or pseudoprogression.

In all patients, 5-ALA (Gliolan) was administered 4 hours before surgery. Two different machines were used for iCT: a small-bore (CereTom, NeuroLogica, USA) and a large-bore (BodyTom Elite, NeuroLogica, USA) portable scanner. A navigated US device (MyLab Twice, Esaote, Italy) was also used. IONM included motor evoked potentials (MEPs) and somatosensory evoked potentials (SSEPs) as well as direct cortical and subcortical electrical stimulation (DES) with monopolar and bipolar probes.

Our multimodal intraoperative protocol has already been described.9,13 After craniotomy and before dural opening, navigated iUS acquisition was performed. All patients underwent neuronavigated 5-ALA fluorescenceguided microsurgical tumor resection. IONM was used when the tumor was near or infiltrated eloquent cortical and subcortical areas. Resection was stopped when DES documented clear responses of eloquent tissue at low-intensity stimulations $(10 \mathrm{~mA})$. When microsurgical resection of all visible fluorescent tissue was deemed complete, navigated iUS was repeated. In cases in which a tumor remnant was clearly visible on iUS images, further resection was performed if it was in a noneloquent area (DES check). After completing iUS and 5-ALA-guided resection, iCT with and without contrast was performed in order to confirm complete resection and the absence of complications. In other cases (remnants not easily visible with iUS because of artifacts), iCT with and without contrast detecting tumor remnants was used to update neuronavigation and to localize and resect those remnants if located in safe areas.

Postoperative radiological evaluation was performed using MRI with and without Gd within 48 hours after surgery.

Volumetric evaluation of EOTR was expressed as a percentage. Preoperative and postoperative Gd-enhanced T1-weighted MRI sequences were used to measure the rate of tumor resection. Tumor volumes were obtained by manual segmentation and a volume rendering method, using a dedicated tool of the StealthViz planning station. EOTR was then calculated using the formula proposed by Sanai and Berger: ${ }^{14}$ [(preoperative tumor volume - postoperative tumor volume)/preoperative tumor volume] $\times 100$. In all patients, pre- and postoperative KPS scores were evaluated. PFS was calculated from the date of surgery to the date of recorded evidence of disease progression, according to the Response Assessment in Neuro-Oncology (RANO) criteria; OS was calculated from the second surgery date to the death date or to March 2020 (i.e., last follow-up).

\section{Results}

Twenty patients (12 male [60\%]), with a mean age of 54.5 years (range $12-78$ years), underwent second surgery 
TABLE 1. Demographic and clinical features of 20 patients who underwent a second surgery for recurrent HGG

\begin{tabular}{lc}
\hline \multicolumn{1}{c}{ Characteristic } & Value \\
\hline Sex & 12 \\
$M$ & 8 \\
$F$ & \\
Age in yrs & 54.5 \\
Mean & $12-78$ \\
Range & \\
Tumor location & $4 / 20$ \\
Noneloquent brain & $8 / 20$ \\
Near eloquent brain & $8 / 20$ \\
Eloquent brain & 19.1 \\
PFS after 1st surgery in mos & $2-108$ \\
Mean & \\
Range & 7.7 \\
Time to 2nd progression in mos & $2-11$ \\
Mean & \\
Range & 25.4 \\
OS in mos (excluding 2 long survivors) & $10-52$ \\
Mean & \\
Range &
\end{tabular}

for recurrent HGG (Table 1). The mean time between the first and second surgeries was 19.1 months (range 2-108 months). Histopathological analysis at the first surgery revealed 2 AAs and 18 GBMs. In 5 cases, the first surgery was performed at another institution. Only 2 patients did not undergo chemotherapy or radiotherapy between the first and second surgeries. The recurrent tumor functional grades $^{12}$ were 1 (noneloquent area), 2 (near an eloquent area), and 3 (eloquent area) in 4, 8, and 8 cases, respectively. In the latter cases, we preferred IONM in an asleep fashion to preserve motor function because language and the metacognitive aspect were already impaired by pathol- ogy, previous surgery, radiotherapy, and drugs. In these cases, awake surgery was not feasible, and sometimes the resolution of mass effect in the asleep mode allows clinical improvement in language and the metacognitive aspect.

In all but 2 patients $100 \%$ EOTR was obtained. In the other 2 cases, a remnant was intentionally left behind because it was close to the primary motor area. Resection was stopped when positive responses to subcortical stimulation at a $10-\mathrm{mA}$ intensity were found.

During tumor resection, 5-ALA fluorescence was consistently seen in the peritumoral area because of tumoral infiltration (FLAIR-hyperintense regions) and reactive gliosis, as revealed by histopathological analysis of the targeted samples (Fig. 1).

All iUS images, including the one obtained before opening the dura, were altered by the presence of artifacts and guardedly interpreted in all cases. In particular, when using iUS in recurrent HGGs, we could not differentiate among the hyperechoic signal of "solid" tumor (due to hypercellularity), the hypoechoic signal of the cysticnecrotic core, and the weakly hyperechoic signal of the surrounding area because of the presence of edema and infiltrative tumor, although these signals are usually observed in primary HGG. ${ }^{5,15,16}$ Conversely, we consistently found a nonhomogeneous signal characterized by alternation of the hyperechoic and hypoechoic areas due to the presence of radiation-altered tissue, gliosis, and recurrent tumor (Fig. 2). Moreover, combination with MR (i.e., navigated iUS) documented the presence of hyperechoic areas also in apparently "normal" brain regions (Fig. 2 yellow arrow).

iCT detected tumor remnants in 3 cases, but further resection was performed in only 1 patient (Fig. 3). Six patients (30\%) experienced worsening of their neurological conditions: 4 recovered within 90 days, 1 partially recovered, and 1 was left with permanent left hemiplegia. In the last patient, intraoperative MEPs showed 64\% signal reduction.
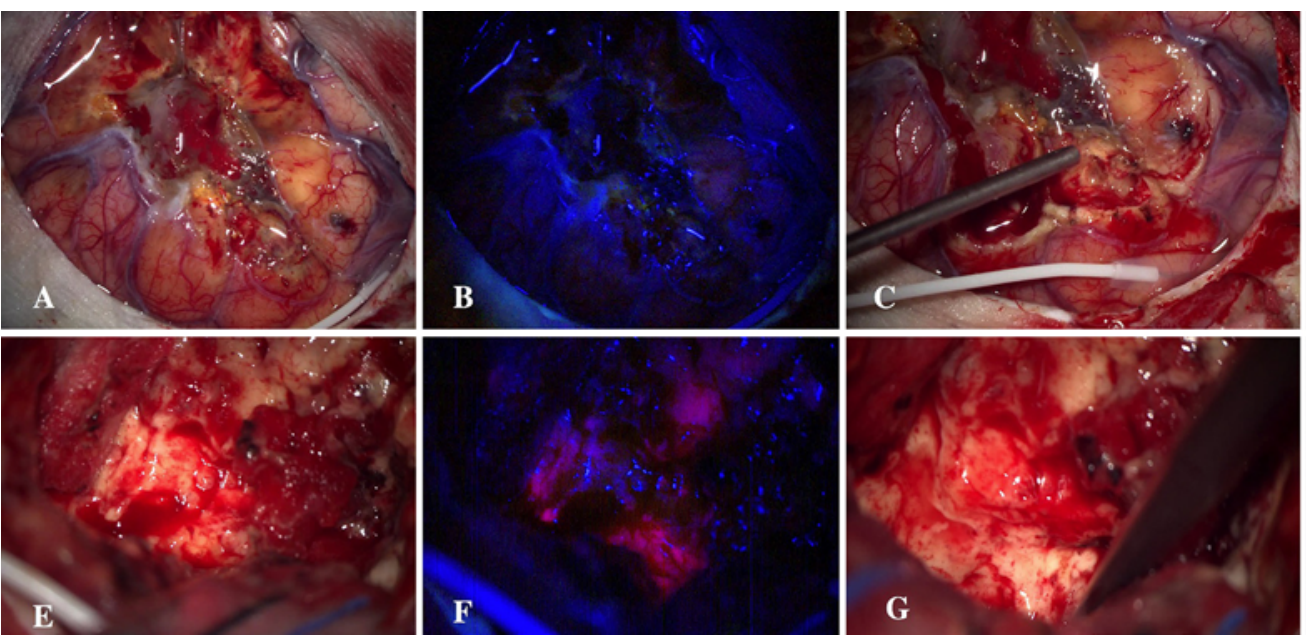

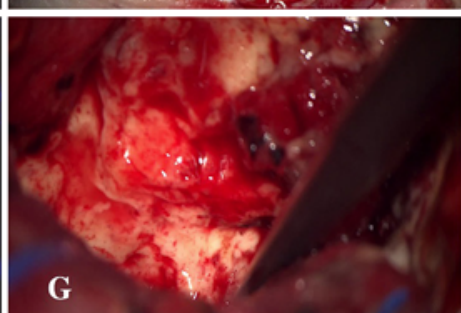

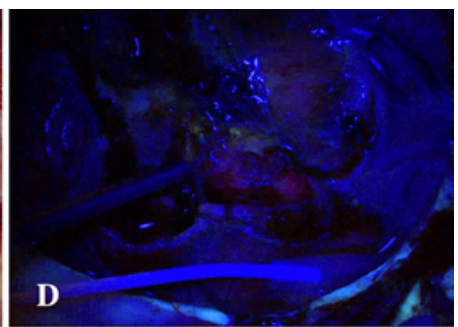

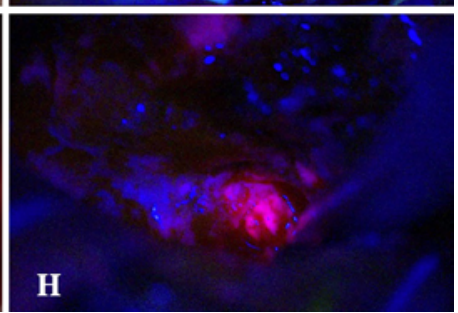

FIG. 1. Comparison between white light and blue light showing the efficacy of 5-ALA to detect tumor in a recurrence. Clear pathological tissue without fluorescence (A). Scarring and radionecrosis (B). Tissue (C) immediately visible under scarring, with blue light fluorescence $(D)$. In the other images $(E-H)$, the tumor core appears with a lava-like fluorescence, and the peripheral tumoral areas with tumoral cell infiltration and gliosis appear with faint fluorescence. 


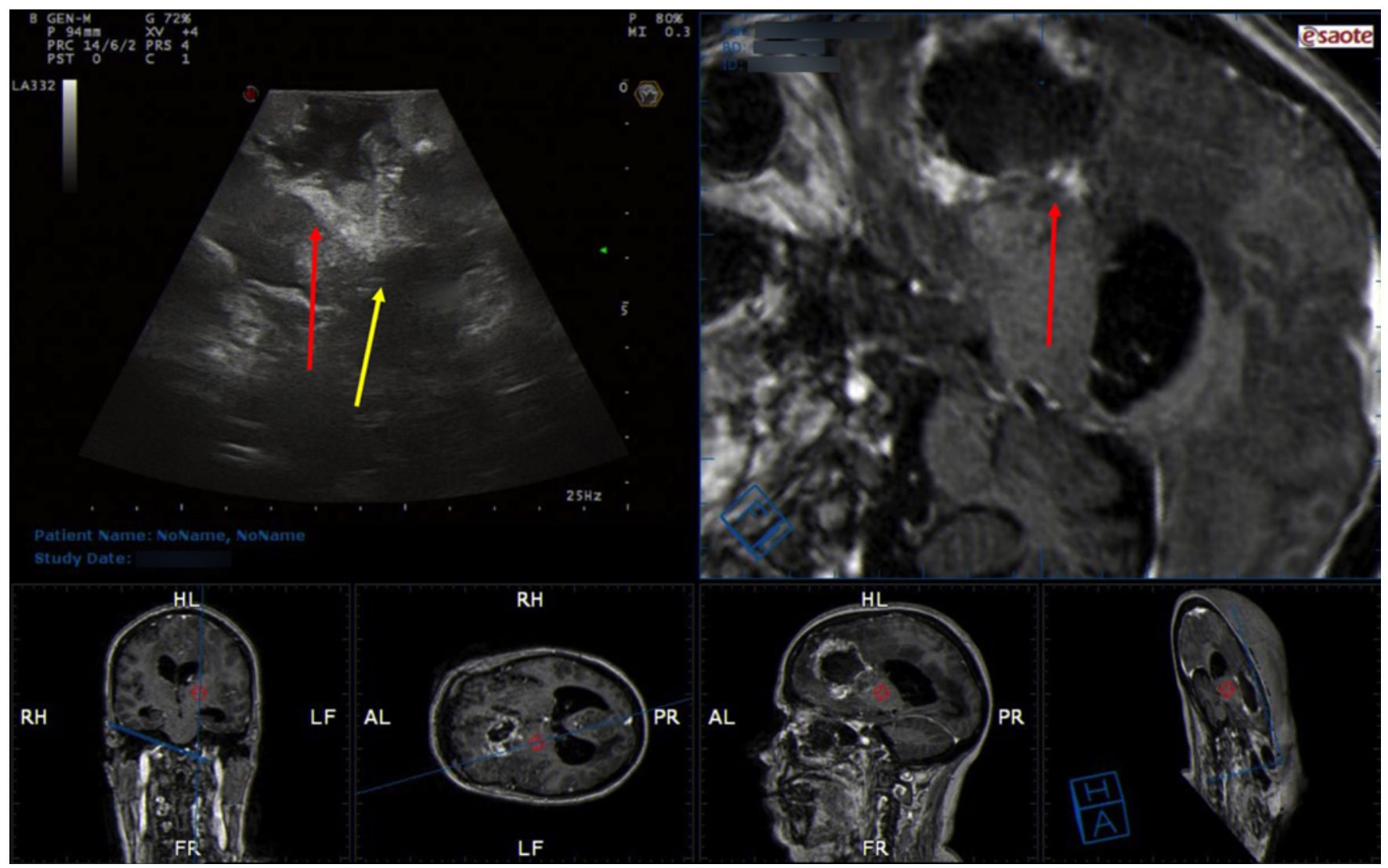

FIG. 2. Recurrent frontal GBM. iUS (left) performed before opening the dura documented a hyperechoic signal in the bottom of the surgical field, whereas navigated MRI sequences (right and lower) showed no tumor infiltration (red arrows). Moreover, posterior acoustic shadowing hides underlying areas (yellow arrow).

The KPS score changed from a median preoperative value of 80 (SD 12.3) to a median postoperative value of 75 (SD 19.5). But at the last follow-up, the mean KPS score was 70 (SD 10.0). The distribution and variation of the KPS scores are graphically depicted in Fig. 4.

The mean PFS after the first surgery was 19.1 months (range 2-108 months); after the re-resection, it was 7.7 months (range 2-11 months). The mean OS was 25.4 months (range 10-52 months), excluding 2 long survivors (120 and 80 months). Two patients died within 60 days after surgery because of general complications, and 3 patients were still under follow-up at the end of the study.

\section{Discussion}

The PFS rates after diagnosis of and surgery for HGG are $11 \%$ and $4 \%$ at 2 and 5 years, respectively. ${ }^{4}$ After radiotherapy, the initial recurrence site is located a few centimeters from the first surgical field or inside it. ${ }^{17-19}$ However, diagnosis of a recurrence on follow-up MRI can be challenging: $20 \%$ of patients develop pseudoprogression after chemoradiotherapy, ${ }^{20}$ while after the use of antiangiogenic agents the tumor may be less apparent (pseudoresponse). In the event of HGG recurrence, no worldwide consensus exists concerning its treatment. The current literature suggests surgery together with radiotherapy or chemoradiotherapy: ${ }^{5,21-23}$ re-resection results in both an updated diagnosis and therapeutic treatment. Brain tissue alterations and bad healing processes after radiotherapy, together with extension of resection to the borders of functional areas at the first surgery, are usually the two main disadvantages of redo surgery. Moreover, a postoperative (i.e., after the second surgery) higher rate of neurological deficits, with a subsequent reduction in quality of life, is reported as a predictive factor for poor survival. ${ }^{24,25}$

\section{Number of Resections and Survival}

Kirkpatrick and Sampson reviewed different treatment strategies and reported redo surgery as a valuable treatment option, even if consistent benefit was not clear. ${ }^{26}$ And although some studies have reported their worst survival rates after reoperation, ${ }^{27}$ more recent surgical experience has shown an increase in survival following multiple resections..$^{21,22}$ Chaichana et al. reported an improvement in OS for patients undergoing multiple resections for GBM recurrences, experiencing a total survival range from 6.8 months for 1 reoperation to 26.6 months after 4 reoperations. ${ }^{6}$

\section{Patient Selection for Surgery}

Considering the wide treatment options for HGG recurrences as well as the surgical risks, patient selection criteria for surgery should be as strict as possible. Thus, the choice of surgery follows correct patient selection. In 2008 Bar- 

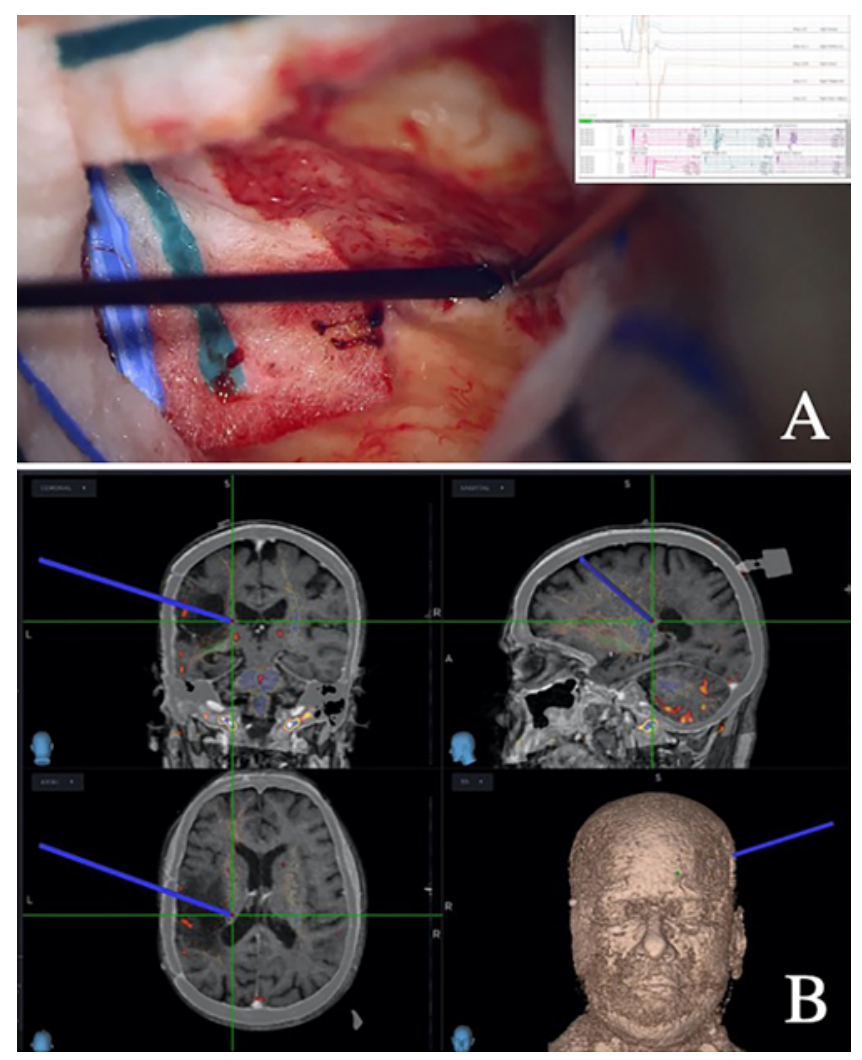

FIG. 3. Multimodal protocol based on different technologies. A monopolar probe is used for subcortical stimulation, detecting a positive response in the deepest portion of the surgical cavity $(\mathbf{A})$. The precise location of this point is localized by a navigation probe $(A)$ and verified by neuronavigation based on merged preoperative MR and iCT images (B).

bagallo et al. reported that the ideal patient is younger than 50 years of age (even though older patients can be treated), with a KPS score $\geq 60$, bearing a resectable tumor (at least $98 \%$ of tumor volume) located in easily accessible and noneloquent areas, and with a minimum 6-month interval between diagnosis and first recurrence. ${ }^{28}$ In 2010 Park et al. proposed a scoring system with the intent to predict survival after surgery for glioma recurrence. In this scale the factors associated with poor postoperative survival were tumor involvement of eloquent areas, KPS score $\leq 80$, and tumor volume $\geq 50 \mathrm{~cm}^{3} .{ }^{29}$ Age has also been considered a prognostic factor in some studies,, 30 whereas in a more recent paper Chaichana et al. did not find a significant correlation between age and prognosis. ${ }^{6}$ Thus, just as in a first surgery, HGGs recurring even in elderly patients can be treated surgically. Age can be considered a prognostic factor if we assume that it is easier in older people to find neurological deficits or other general impairments unrelated to the tumor itself. In these cases, quality of life would be lower after surgery, but not because of surgical damage.

\section{New Focal Deficits in Recurrent Tumor Surgery}

The incidence of neurological deficits after reoperation is higher than after a first surgery. The main causes are brain alterations after a first surgery, radiotherapy, and functional resection margins already reached during the

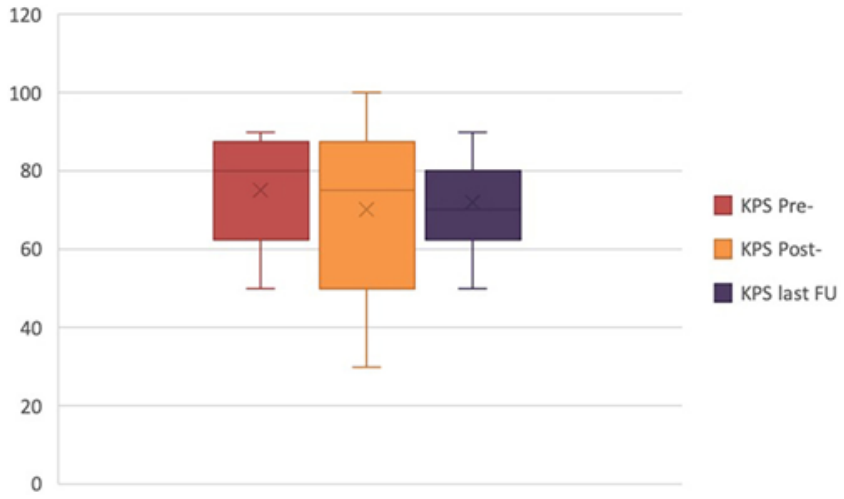

FIG. 4. Boxplot showing the KPS score variation before and after surgery and at the final follow-up.

first surgery. Therefore, IONM plays a key role for recurrent tumors. In their study, Oppenlander et al. found that 84 of 170 patients harbored tumors in eloquent areas and required motor and/or speech mapping during surgery. ${ }^{5}$ Patients' early postoperative neurological profiles were evaluated using the National Institutes of Health Stroke Scale (NIHSS): deterioration at 7 days after surgery was indicated by scores of $0.53 \pm 2.07$ and $1.27 \pm 2.71$ for EOTRs $\leq 80 \%$ and $\geq 95 \%$, respectively. Similarly, at 30 days, for the same EOTR values, the NIHSS score varied by $0.3 \pm 2.02$ and $0.77 \pm 2.32$, respectively. Interestingly, after 1 month, differences in NIHSS score deterioration were not significant with respect to EOTR.

\section{Impact of EOTR on Survival Rate}

Survival differences have been noted not only for number of resections but also for different EOTR rates. Bloch et al. demonstrated that EOTR at the first surgery greatly influences OS, as compared to the resection rate at the second surgery. ${ }^{21}$ In particular, GTR at the first surgery followed by GTR and subtotal resection (STR) at the second surgery resulted in median survival rates of 20.4 and 18.4 months, respectively. In contrast, STR at the first surgery followed by GTR and STR at the second surgery provided median survival rates of 19 and 15.9 months, respectively. Therefore, GTR at the second surgery overcomes the negative impact of incomplete resection at the first surgery. Conversely, a worse clinical outcome results from two consecutive incomplete resections.

\section{5-ALA Fluorescence in Recurrent Tumor Surgery}

As for primary HGG surgery, different intraoperative tools have been introduced to improve the extent and safety of resection for recurrences.

Although the use of 5-ALA fluorescence to guide tumor resection is well documented for first surgeries for HGGs, Lau et al. reported low specificity: $62.3 \%$ of nonfluorescent biopsies included histologically proven tumor tissue and $35.4 \%$ of fluorescent samples were not infiltrated. ${ }^{31} \mathrm{In}$ recurrent glioma, the false-positive rate increases because of inflammatory cells and reactive astrocytes secondary to prior surgery and radiotherapy. Utsuki et al. analyzed a series of histological samples from HGG surgeries and 
found that 5 of 11 recurrent gliomas were false positive for 5-ALA fluorescence. ${ }^{32}$ Moreover, Nabavi et al. reported that areas of infiltration in recurrent glioma showed a different fluorescence pattern with regard to similar areas of infiltration in primary glioma surgery. ${ }^{48}$ Indeed, during the second surgery, fluorescence was alternatively weak and strong and not uniform as in primary surgery because areas of tumoral proliferation could be masked by scarring and radionecrosis. ${ }^{33-35}$ However, resection involving all fluorescent tissue, but still respecting intraoperative brain mapping data, is associated with a higher survival rate. ${ }^{36}$ When such a strategy is applied, the incidence of focal neurological deficits ranges from $6.2 \%$ to $11 \% .^{36,37}$ In ex novo HGG on primary diagnosis, we have demonstrated the role of FLAIRectomy (i.e., supramarginal resection based on 3D FLAIR sequences) in survival. ${ }^{38}$ The same target could be pursued on recurrent tumors, with special attention to achieving a maximal safe resection. The surrounding FLAIR hyperintensity zone in recurrent HGG is due to infiltrative neoplastic cells, edema, gliosis related to previous surgery, and effects secondary to radiotherapy. It is also well known that the neuroplasticity in HGG patients is lower than that in patients affected by low-grade glioma. ${ }^{39}$ Indeed, we emphasize that aggressive resection is feasible, but it is crucial to stop surgery within the functional boundaries.

\section{iUS in Recurrent Glioma Surgery}

Several studies have reported that the use of US in primary glioma surgery improves the EOTR.$^{15,40,41}$ Moreover, new technical tools, such as contrast-enhanced US, ${ }^{16,42}$ miniaturized probes, and liquids other than normal saline or Ringer's solution, ${ }^{43}$ are under investigation to improve the quality of iUS imaging. Accepted advantages of iUS are its fast and easy use, its low cost, and the possibility of integrating and merging it with preoperative MRI and neuronavigation; however, $\mathrm{B}$-mode or $\mathrm{BD}$ iUS is also useful to correct for brain shift. Nonetheless, several factors, such as artifacts due to blood, the size and shape of the surgical cavity, the depth of the lesion, and surgical manipulation, can reduce iUS sensitivity to localize tumor remnants. In particular, Renovanz et al. reported that in recurrent HGG, artifacts and barriers to US penetration are also caused by radiotherapy-associated alterations. ${ }^{44}$

\section{iMRI in Recurrent HGG Surgery}

MRI is currently considered a safe and effective intraoperative imaging tool in primary glioma surgery. QuickWeller et al. ${ }^{45}$ reported their experience with iMRI and 5-ALA fluorescence-guided resection in glioma recurrences; they found positive areas of tumor infiltration on iMRI after all 5-ALA fluorescent tissue had been resected. Indeed, after continued resection of those infiltrated areas, histopathological analysis confirmed the presence of neoplastic cells. However, as in primary tumor surgery, iMRI has advantages and disadvantages to be considered. iMRI is highly reliable for visualization of brain parenchyma and tumor remnants in primary surgery, but its specificity in recurrent cases is lower because of artifacts secondary to radionecrosis and previous surgical manipulation. Moreover, iMRI machines are expensive, and the entire operating room as well as surgical instruments must be MRI compatible, further increasing the costs associated with such technology. However, obtaining an iMRI scan takes about 1 hour, ${ }^{46}$ and patient positioning can be challenging in specific tumor localizations (i.e., occipital) ${ }^{45}$

\section{ICT in Recurrent HGG Surgery}

CT is a safe and effective intraoperative tool and cheaper than iMRI for glioma surgery. ${ }^{9}$ Its ionizing radiation dose and lower accuracy in brain visualization have limited its use in the past. However, our experience with $\mathrm{iCT}$ in glioma surgery confirms that it is a safe and effective imaging tool during HGG surgery. ${ }^{9,47}$ The cost of iCT equipment and the scan time are lower than those associated with iMRI, and there is no need for dedicated surgical tools in the operating room, except for radiolucent skull clamps. iCT images are used to update neuronavigation systems and perform real-time navigation. The use of iCT is also feasible in awake craniotomies. ${ }^{13}$ iCT is particularly useful to identify tumor remnants, by comparison of contrast-enhanced pre- and intraoperative (postresection) scans, and to rule out early postoperative complications such as hemorrhage. ${ }^{9}$

\section{Integration of Intraoperative Tools in Recurrent HGG Surgery}

While neuronavigation is a mainstay of recurrent tumor surgery, 5-ALA fluorescence is less specific in a second surgery than in the primary surgery. As reported above, even in our cohort, 5-ALA fluorescence was influenced by previous healing processes and radiotherapy-induced damage. In all patients, fluorescence in the tumor edges, as well as in deep tumor infiltration, was neither as clear nor as evident as in the primary glioma surgery. iCT can help to differentiate between tumor and other parenchymal alterations. In 3 of our cases the iCT scan detected tumor remnants; however, surgery was performed in only 1 case because tumors in the others were located in eloquent areas. Conversely, iUS was not reliable: previous surgical manipulation and radionecrosis were sources of false positives. The relevant hyperechoic and hypoechoic areas could be mistakenly interpreted as tumor infiltration (Fig. 2) and could have covered the underlying parenchyma, eventually reducing the sensitivity of the technique. In our series, we constantly observed the presence of artifacts affecting image interpretation since the first image acquisition before dural opening. Limitations in the reliability of US as intraoperative guidance to resect HGG were also confirmed by integration with neuronavigation (i.e., navigated iUS), which demonstrated the presence of questionable hyperechoic areas also in apparently "normal" brain regions (Fig. 2). Unless miniaturized probes are used, several limitations of iUS application in recurrent glioma cases need to be overcome. We also integrated iMRI-based navigation with ${ }^{11} \mathrm{C}$-MET-PET images merged with MRI. This was useful to guide and focus the microsurgical resection on more metabolically active areas of the recurrent tumor, independently from the presence of Gd-enhancing areas on T1-weighted MRI (Fig. 5B and C).

Conversely, important information comes from IONM. 

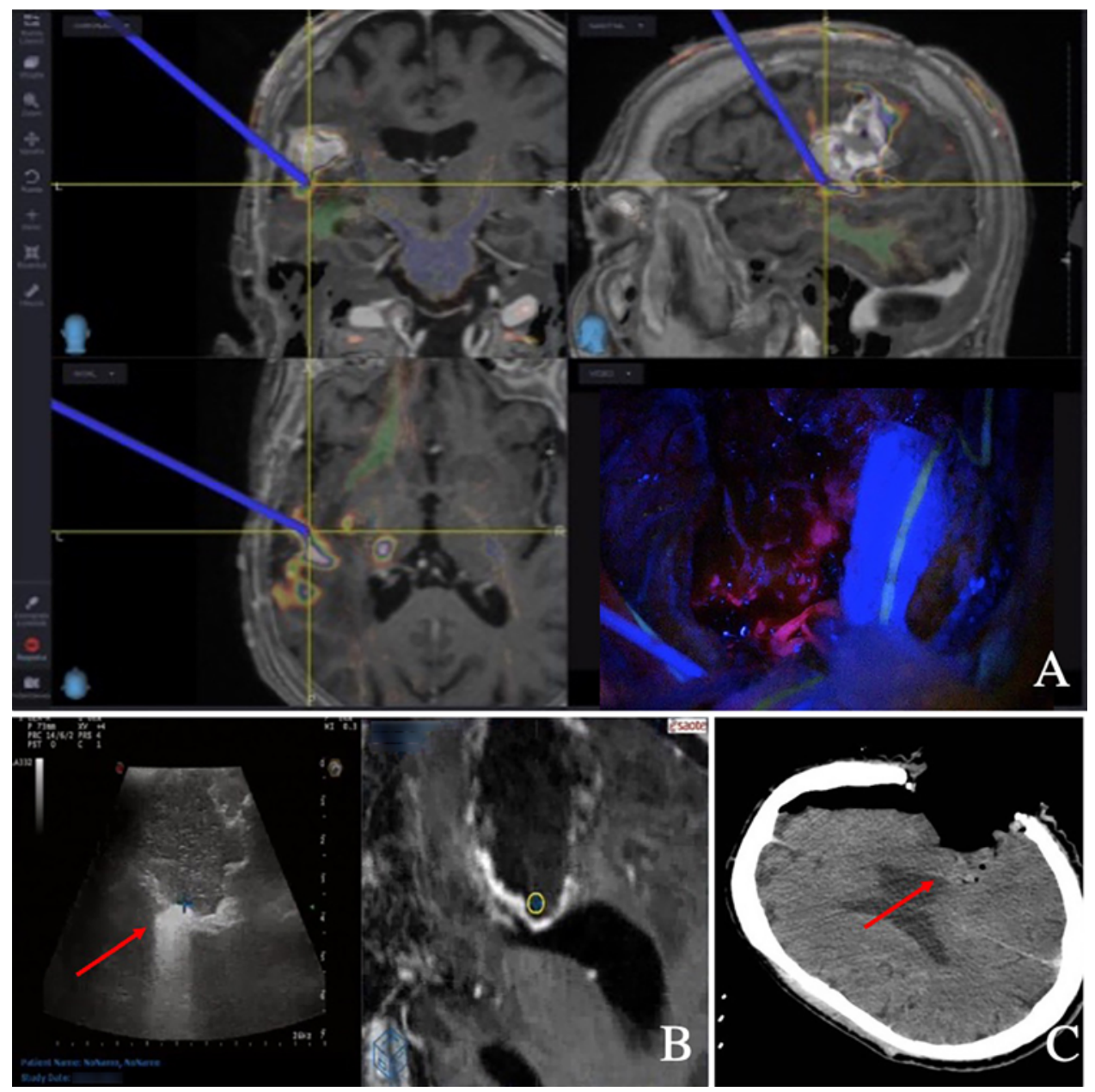

FIG. 5. Neuronavigation was based on Gd-enhanced T1-weighted sequences, and ${ }^{11} \mathrm{C}-\mathrm{MET}$-PET was used to identify tumor nodules with higher metabolism. Such findings were also confirmed by the presence of 5-ALA fluorescent tissue in the same area (A). At the end of iUS and 5-ALA fluorescence-based resection, a small nodule was identified (red arrow, B). This tumor remnant was intentionally left, as demonstrated by iCT (red arrow, C) to avoid damage to the corticospinal tract.

Because functional edges or eloquent areas can be determined during both the first surgery and the recurrence surgery, functional mapping can help to avoid postoperative neurological deficits. In particular, even if few MEP variations are not directly associated with neurological deficits, in 1 patient a wide drop in MEP potential was related to a left upper-limb complete motor deficit. All but 2 patients had no neurological deficits at 90 days after surgery. Using multiple intraoperative tools, we obtained $100 \%$ EOTR in all but 2 cases, providing a long survival (25.4 months) in all but 2 patients, who died because of general complications. Kaplan-Meier curves documenting the time to second progression and the OS of the cohort are shown in Fig. 6.

\section{Study Limitations}

Our study has some limitations, including the small number of patients and the absence of information on and correlations with the tumor molecular features of each patient. Nevertheless, the aim of this paper was not focused on pathology, but on the operative technology used to approach it independently of its biological behavior.
This is the reason we have not specified the molecular features of tumors-and because it is well known that EOTR has an impact on OS independent of tumoral molecular biology.

We would like to open the way for the study of future perspectives related to the technological advances and possibilities in HGG recurrence surgery. We would like to show the advantages and disadvantages of intraoperative tools, helping surgeons to better identify tumor tissue and distinguish it from scar tissue, radionecrosis, and other tissue modifications due to previous treatments.

\section{Conclusions}

This is the first study reporting the integration of navigated microsurgical resection under 5-ALA fluorescence guidance coupled with iUS, iCT, and IONM for glioma recurrences. A safe and wide-as-possible EOTR can be reached in almost all patients using this protocol, improving life expectancy and overcoming technical difficulties related to brain tissue artifacts secondary to complementary therapies performed after the first surgery. 


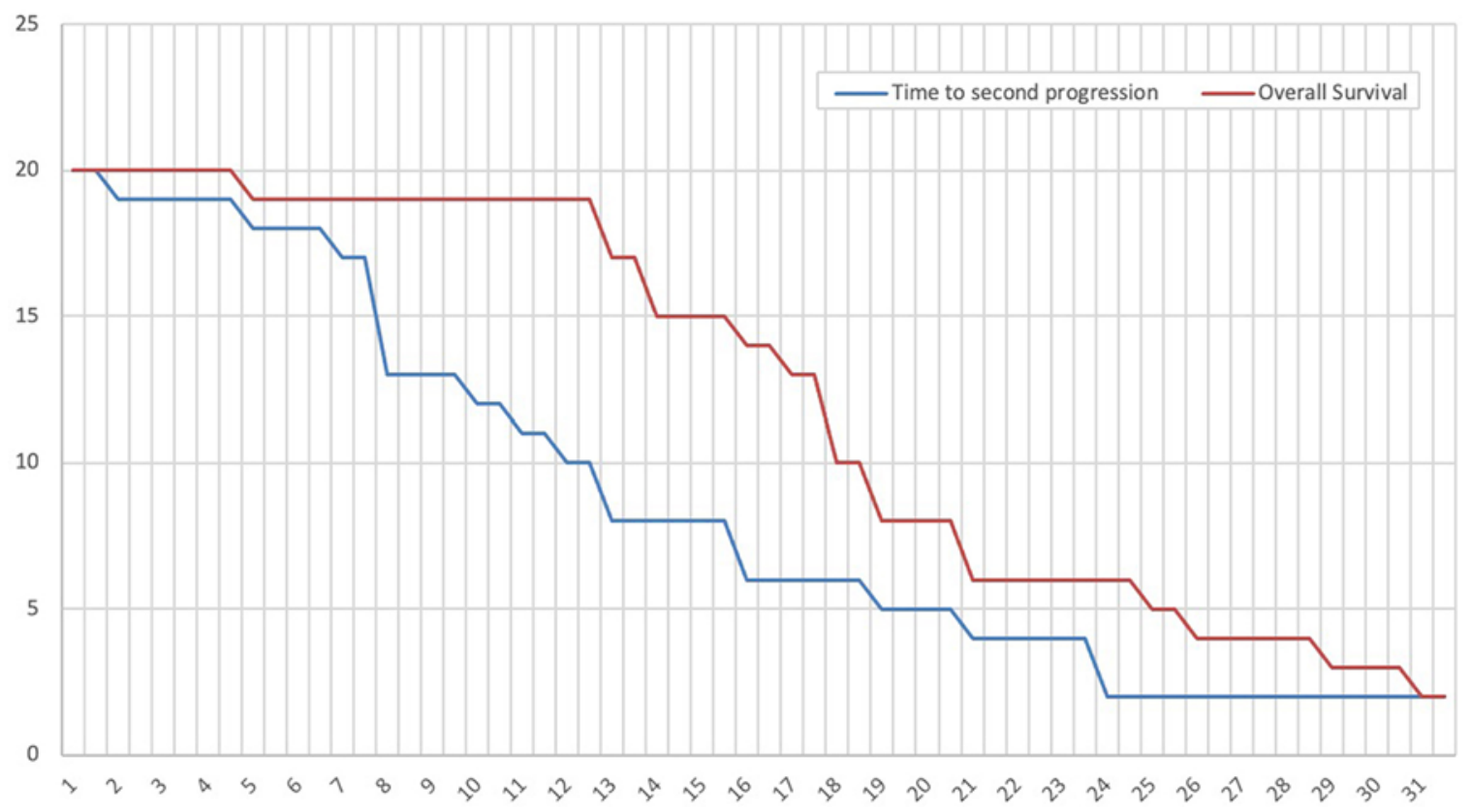

FIG. 6. Kaplan-Meier curves documenting the time to second progression and the OS of the cohort. The x-axis represents the number of months and the $y$-axis the number of patients.

Although this is a pilot study and further experience is needed to confirm our early results, we submit that the use of this integrated multimodal surgical protocol can offer maximal safe resection even in $\mathrm{HGG}$ recurrence.

\section{Acknowledgments}

We thank the Scientific Bureau of the University of Catania for language support.

\section{References}

1. Ostrom QT, Gittleman H, Liao P, et al. CBTRUS Statistical Report: primary brain and other central nervous system tumors diagnosed in the United States in 2010-2014. Neuro Oncol. 2017;19(suppl 5):v1-v88.

2. Rudà R, Houillier C, Maschio M, et al. Effectiveness and tolerability of lacosamide as add-on therapy in patients with brain tumor-related epilepsy: results from a prospective, noninterventional study in European clinical practice (VIBES). Epilepsia. 2020;61(4):647-656.

3. Altieri R, Zenga F, Ducati A, et al. Tumor location and patient age predict biological signatures of high-grade gliomas. Neurosurg Rev. 2018;41:599-604.

4. Stupp R, Hegi ME, Mason WP, et al. Effects of radiotherapy with concomitant and adjuvant temozolomide versus radiotherapy alone on survival in glioblastoma in a randomised phase III study: 5-year analysis of the EORTC-NCIC trial. Lancet Oncol. 2009;10(5):459-466.

5. Oppenlander ME, Wolf AB, Snyder LA, et al. An extent of resection threshold for recurrent glioblastoma and its risk for neurological morbidity. J Neurosurg. 2014;120(4):846-853.

6. Chaichana KL, Zadnik P, Weingart JD, et al. Multiple resections for patients with glioblastoma: prolonging survival. $J$ Neurosurg. 2013;118(4):812-820.

7. Sastry R, Bi WL, Pieper S, et al. Applications of ultrasound in the resection of brain tumors. J Neuroimaging. 2017;27(1):5-15.

8. Hosoda T, Takeuchi H, Hashimoto N, et al. Usefulness of in- traoperative computed tomography in surgery for low-grade gliomas: a comparative study between two series without and with intraoperative computed tomography. Neurol Med Chir (Tokyo). 2011;51(7):490-495.

9. Barbagallo GMV, Palmucci S, Visocchi M, et al. Portable intraoperative computed tomography scan in image-guided surgery for brain high-grade gliomas: analysis of technical feasibility and impact on extent of tumor resection. Oper Neurosurg (Hagerstown). 2016;12(1):19-30.

10. Lakomkin N, Hadjipanayis CG. Fluorescence-guided surgery for high-grade gliomas. J Surg Oncol. 2018;118(2):356-361.

11. Motomura K, Natsume A, Iijima K, et al. Surgical benefits of combined awake craniotomy and intraoperative magnetic resonance imaging for gliomas associated with eloquent areas. J Neurosurg. 2017;127(4):790-797.

12. Li YM, Suki D, Hess K, Sawaya R. The influence of maximum safe resection of glioblastoma on survival in 1229 patients: can we do better than gross-total resection? J Neurosurg. 2016;124(4):977-988.

13. Barbagallo GMV, Morrone A, Certo F. Intraoperative computed tomography and awake craniotomy: a useful and safe combination in brain surgery. World Neurosurg. 2018;119: e159-e166.

14. Sanai N, Berger MS. Glioma extent of resection and its impact on patient outcome. Neurosurgery. 2008;62(4):753764, 264-266.

15. Altieri R, Melcarne A, Di Perna G, et al. Intra-operative ultrasound: tips and tricks for making the most in neurosurgery. Surg Technol Int. 2018;33:353-360.

16. Della Pepa GM, Ius T, La Rocca G, et al. 5-Aminolevulinic acid and contrast-enhanced ultrasound: the combination of the two techniques to optimize the extent of resection in glioblastoma surgery. Neurosurgery. 2020;86(6):E529-E540.

17. Wallner KE, Galicich JH, Krol G, et al. Patterns of failure following treatment for glioblastoma multiforme and anaplastic astrocytoma. Int J Radiat Oncol Biol Phys. 1989;16(6): 1405-1409.

18. Lee SW, Fraass BA, Marsh LH, et al. Patterns of failure fol- 
lowing high-dose 3-D conformal radiotherapy for high-grade astrocytomas: a quantitative dosimetric study. Int J Radiat Oncol Biol Phys. 1999;43(1):79-88.

19. Hess CF, Schaaf JC, Kortmann RD, et al. Malignant glioma: patterns of failure following individually tailored limited volume irradiation. Radiother Oncol. 1994;30(2):146-149.

20. Brandsma D, Stalpers L, Taal W, et al. Clinical features, mechanisms, and management of pseudoprogression in malignant gliomas. Lancet Oncol. 2008;9(5):453-461.

21. Bloch O, Han SJ, Cha S, et al. Impact of extent of resection for recurrent glioblastoma on overall survival: clinical article. J Neurosurg. 2012;117(6):1032-1038.

22. Blumenthal DT, Kanner AA, Aizenstein O, et al. Surgery for recurrent high-grade glioma after treatment with bevacizumab. World Neurosurg. 2018;110:e727-e737.

23. Krivoshapkin A, Gaytan A, Salim N, et al. Repeat resection and intraoperative radiotherapy for malignant gliomas of the brain: a history and review of current techniques. World Neurosurg. 2019;132:356-362.

24. McGirt MJ, Mukherjee D, Chaichana KL, et al. Association of surgically acquired motor and language deficits on overall survival after resection of glioblastoma multiforme. Neurosurgery. 2009;65(3):463-470.

25. Chaichana KL, Chaichana KK, Olivi A, et al. Surgical outcomes for older patients with glioblastoma multiforme: preoperative factors associated with decreased survival. $J$ Neurosurg. 2011;114(3):587-594.

26. Kirkpatrick JP, Sampson JH. Recurrent malignant gliomas. Semin Radiat Oncol. 2014;24(4):289-298.

27. Nieder C, Grosu AL, Molls M. A comparison of treatment results for recurrent malignant gliomas. Cancer Treat Rev. 2000;26(6):397-409.

28. Barbagallo GMV, Jenkinson MD, Brodbelt AR. 'Recurrent' glioblastoma multiforme, when should we reoperate? $\mathrm{Br} J$ Neurosurg. 2008;22(3):452-455.

29. Park JK, Hodges T, Arko L, et al. Scale to predict survival after surgery for recurrent glioblastoma multiforme. J Clin Oncol. 2010;28(24):3838-3843.

30. Birk HS, Han SJ, Butowski NA. Treatment options for recurrent high-grade gliomas. CNS Oncol. 2017;6(1):61-70.

31. Lau D, Hervey-Jumper SL, Chang S, et al. A prospective Phase II clinical trial of 5-aminolevulinic acid to assess the correlation of intraoperative fluorescence intensity and degree of histologic cellularity during resection of high-grade gliomas. J Neurosurg. 2016;124(5):1300-1309.

32. Utsuki S, Oka H, Sato S, et al. Histological examination of false positive tissue resection using 5-aminolevulinic acidinduced fluorescence guidance. Neurol Med Chir (Tokyo). 2007;47(5):210-214.

33. Certo F, Stummer W, Farah JO, et al. Supramarginal resection of glioblastoma: 5-ALA fluorescence, combined intraoperative strategies and correlation with survival. J Neurosurg Sci. 2019;63(6):625-632.

34. La Rocca G, Sabatino G, Menna G, et al. 5-ALA "false positives" in cerebral neurooncology: not all that fluorescences is tumor. A case-based update and literature review. World Neurosurg. 2020;137:187-193.

35. La Rocca G, Barresi V, Sabatino G, et al. 5-ALA falsepositive in anaplastic oligodendroglioma, IDH-mutant and 1p/19q-codeleted. Surg Technol Int. 2020;36:453-456.

36. Hickmann A-K, Nadji-Ohl M, Hopf NJ. Feasibility of fluorescence-guided resection of recurrent gliomas using five-aminolevulinic acid: retrospective analysis of surgical and neurological outcome in 58 patients. J Neurooncol. 2015; 122(1):151-160.

37. Hoover JM, Nwojo M, Puffer R, et al. Surgical outcomes in recurrent glioma: clinical article. J Neurosurg. 2013;118(6): $1224-1231$.
38. Certo F, Altieri R, Maione M, et al. FLAIRectomy in supramarginal resection of glioblastoma correlates with clinical outcome and survival analysis: a prospective, single institution, case series. Oper Neurosurg (Hagerstown). Published online October 9, 2020. doi:10.1093/ons/opaa293

39. Sacko O, Lauwers-Cances V, Brauge D, et al. Awake craniotomy vs surgery under general anesthesia for resection of supratentorial lesions. Neurosurgery. 2011;68(5):1192-1199.

40. Selbekk T, Jakola AS, Solheim O, et al. Ultrasound imaging in neurosurgery: approaches to minimize surgically induced image artefacts for improved resection control. Acta Neurochir (Wien). 2013;155(6):973-980.

41. Altieri R, Meneghini S, Agnoletti A, et al. Intraoperative ultrasound and 5-ALA: the two faces of the same medal? $J$ Neurosurg Sci. 2019;63(3):258-264.

42. Prada F, Bene MD, Fornaro R, et al. Identification of residual tumor with intraoperative contrast-enhanced ultrasound during glioblastoma resection. Neurosurg Focus. 2016;40(3):E7.

43. Petridis AK, Anokhin M, Vavruska J, et al. The value of intraoperative sonography in low grade glioma surgery. Clin Neurol Neurosurg. 2015;131:64-68.

44. Renovanz M, Hickmann A-K, Henkel C, et al. Navigated versus non-navigated intraoperative ultrasound: is there any impact on the extent of resection of high-grade gliomas? A retrospective clinical analysis. J Neurol Surg A Cent Eur Neurosurg. 2014;75(3):224-230.

45. Quick-Weller J, Lescher S, Forster MT, et al. Combination of 5-ALA and iMRI in re-resection of recurrent glioblastoma. Br J Neurosurg. 2016;30(3):313-317.

46. Senft C, Bink A, Franz K, et al. Intraoperative MRI guidance and extent of resection in glioma surgery: a randomised, controlled trial. Lancet Oncol. 2011;12(11):997-1003.

47. Barbagallo G, Maione M, Peschillo S, et al. Intraoperative computed tomography, navigated ultrasound, 5-amino-levulinic acid fluorescence and neuromonitoring in brain tumor surgery: overtreatment or useful tool combination? J Neurosurg Sci. Published online July 11, 2019. doi:10.23736/S03905616.19.04735-0

48. Nabavi A, Thurm H, Zountsas B, et al. Five-aminolevulinic acid for fluorescence-guided resection of recurrent malignant gliomas: a phase ii study. Neurosurgery. 2009;65(6):1070-1077.

\section{Disclosures}

The authors report no conflict of interest concerning the materials or methods used in this study or the findings specified in this paper.

\section{Author Contributions}

Conception and design: Barbagallo. Acquisition of data: Di Gregorio, Altieri. Analysis and interpretation of data: Garozzo, Peschillo. Drafting the article: Maione, Garozzo, Altieri. Critically revising the article: Certo, Altieri. Statistical analysis: Maione. Administrative/technical/material support: Certo, Peschillo. Study supervision: Barbagallo, Peschillo.

\section{Supplemental Information \\ Online-Only Content}

Supplemental material is available online.

Supplemental Figures 1 and 2. https://thejns.org/doi/suppl/ 10.3171/2020.10.FOCUS20744.

\section{Correspondence}

Giuseppe Maria Vincenzo Barbagallo: Policlinico "G. Rodolico-S. Marco,” University Hospital, Catania, Italy. gbarbagallo@ unict.it. 